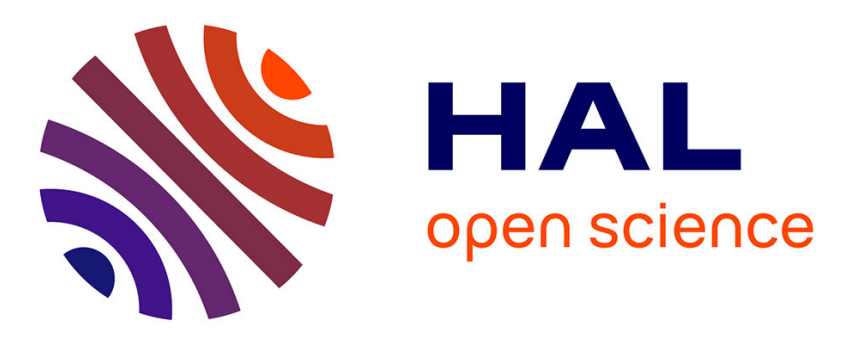

\title{
A Reconfiguration Strategy for Reconfigurable Cable-Driven Parallel Robots
}

Lorenzo Gagliardini, Stéphane Caro, Marc Gouttefarde, Alexis Girin

\section{To cite this version:}

Lorenzo Gagliardini, Stéphane Caro, Marc Gouttefarde, Alexis Girin. A Reconfiguration Strategy for Reconfigurable Cable-Driven Parallel Robots. ICRA: International Conference on Robotics and Automation, May 2015, Seattle, WA, United States. pp.1613-1620, 10.1109/ICRA.2015.7139404. lirmm-01221409

\section{HAL Id: lirmm-01221409 \\ https://hal-lirmm.ccsd.cnrs.fr/lirmm-01221409}

Submitted on 1 Dec 2018

HAL is a multi-disciplinary open access archive for the deposit and dissemination of scientific research documents, whether they are published or not. The documents may come from teaching and research institutions in France or abroad, or from public or private research centers.
L'archive ouverte pluridisciplinaire HAL, est destinée au dépôt et à la diffusion de documents scientifiques de niveau recherche, publiés ou non, émanant des établissements d'enseignement et de recherche français ou étrangers, des laboratoires publics ou privés. 


\title{
A Reconfiguration Strategy for Reconfigurable Cable-Driven Parallel Robots
}

\author{
Lorenzo Gagliardini $^{1}$, Stéphane Caro $^{2}$, Marc Gouttefarde ${ }^{3}$ and Alexis Girin ${ }^{1}$
}

\begin{abstract}
This paper deals with Reconfigurable CableDriven Parallel Robots (RCDPRs). A RCDPR is able to change the locations of its cable exit points, the latter being defined as the connection points between the cables and the robot base frame. Given a RCDPR, a set of possible reconfigurations, a desired platform path and a description of the robot environment, the reconfiguration strategy proposed in this paper selects the optimal configurations to be associated to each point of the desired path. The selection of the optimal configurations can be performed with respect to several criteria such as the number of configuration changes, the number of cable reconfigurations and the robot stiffness. In this paper, the optimization is performed using a Dijkstra's based algorithm.
\end{abstract}

\section{INTRODUCTION}

Cable-Driven Parallel Robots (CDPR) are a particular class of parallel robots whose legs consist of cables (Fig. 1 shows an example of CDPR). CDPRs have been widely investigated in the last two decades. They are usually characterised by a large workspace size, their payload capability is quite high and their inertia is relatively low [1]. Therefore, CDPRs can be employed in several applications, which require a combination of the previous characteristics: A successful example is represented by the shooting of sport events [2], [3]. Other applications have been proposed in the construction and logistic sectors, where heavy loads have to be moved [4], [5], [6]. CDPRs can also be used for rehabilitation purposes [7] and as haptic devices [8]. CDPR studies deal also with other applications, such as rescue operations [9], artistic painting [10] and 3D scanning [11].

A drawback of CDPRs is related to the potential collisions between the cables and the surrounding environment. Furthermore, the non-rigid nature of the cables influences the static equilibrium of the robot. In fact, the cables cannot push on the platform and the equilibrium depends notably on the cable layout.

When a task requires high performances, and when the working environment is cluttered, Reconfigurable CableDriven Robots (RCDPR) can provide better performances than standard CDPRs. By reconfigurable we mean that the RCDPR is able to change the locations of its cable

This work is supported by the IRT Jules Verne, Bouguenais, France (Project CAROCA, Evaluation des CApacités de la RObotique à CÂbles dans un contexte industriel)

${ }^{1}$ L. Gagliardini and A. Girin are with Robotics Team, IRT Jules Verne, Chemin du Chaffault, 44340, Bouguenais, France, \{lorenzo.gagliardini, alexis.girin\}@irt-jules-verne.fr

${ }^{2} \mathrm{~S}$. Caro is with CNRS-IRCCyN, 1, rue de la Noë, 44321, Nantes Cedex 03, France, Stephane.Caro@irccyn.ec-nantes.fr

${ }^{3} \mathrm{M}$. Gouttefarde is with the Laboratoire d'Informatique, de Robotique et de Micro-électronique de Montpellier (LIRMM-CNRS-UM), 161, rue Ada, 34392, Montpellier Cedex 05, France, marc.gouttefarde@lirmm.fr

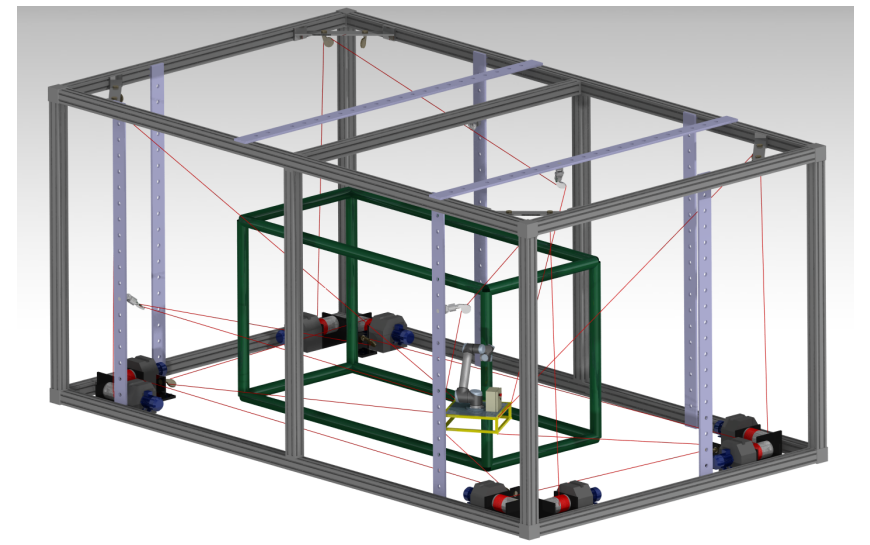

Fig. 1. Example of a RCDPR design. The robot shown in the picture is a concept created in the framework of the CAROCA project whose intent is the painting of tubular structures.

exit points, defined as the connection points between the cables and the robot base frame. Thereby, by changing the cable layout, it should be possible to avoid collisions between the cables and the environment. At the same time, some robot characteristics may be improved: increasing stiffness, augmenting payload capability or decreasing the cable tensions.

The need of performing manufacturing operations over wide products is the main motivation of this work. The reconfiguration strategy proposed in this paper can notably be used to avoid unacceptable cable-environment collisions which usually take place when a non-configurable CDPR is considered.

The first studies on RCDPR have been performed in the frame of the NIST RoboCrane project [4]. More recent investigations have been performed by Rosati et al. [12], as well as by Zhuo et al. [13], Izard et al. [14] and Nguyen et al. [15], [16]. The design of RCDPRs is one of the main goal of the European project CableBot [17].

Rosati et al. [12] proposed a design and a reconfiguration strategy based on an analytical optimal cable layout characterization. This strategy can be applied efficiently to planar robots. However, it can hardly be extended to three-dimensional case studies, because the required cable layout analytical description is unknown for spatial CDPRs. Zhuo et al. [13] proposed to increase the Degrees of Freedom (DoF) of the robot using mobile connection points on the base. Nguyen et al. [15] developed a reconfiguration strategy consisting of two sub-optimization problems. The first sub-problem aims at finding the bounds on the 
reconfiguration parameters with respect to a set of non-linear constraints. The computed bounds can be used in the second sub-problem, which is a box-constrained optimization problem. Nguyen et al. proposed several criteria, related to the cable tensions and the robot energy consumption.

For a RCDPR, several cable configurations may be appropriate to perform a given task. A cable configuration is defined as the positions of the exit points, the positions of the cable connection points on the platform and the layout (arrangement) of the cables between these two sets of points. In the sequel, we suppose that the RCDPR under study can assume a discrete set of cable configurations. Given a desired platform path and the task working conditions, the procedure is able to define the best set of configurations to assign to each point of the desired path. The selection of the optimal configurations is performed using a graph based approach. This approach associates to each point of the path a configuration by minimizing one or several criteria, such as the overall number of cable reconfigurations or the platform positioning error.

This paper is organized as follows. Section II presents the geometric, static and elasto-static models used in this work. Section III defines the main steps of a reconfiguration. Section IV proposes a reconfiguration strategy and details the corresponding algorithm, focusing on Dijkstra's algorithm [18], [19], [20] and on the graph construction procedures. Section $\mathrm{V}$ provides an illustrative example and Section VI concludes this paper.

\section{GEOMETRIC AND ELASTO-STATIC ROBOT MODELS}

A CDPR is composed of a mobile platform connected to a fixed base by $m$ cables. The pose of the platform, $\mathbf{p}$, is described with respect to a fixed absolute frame, $\mathscr{F}_{b}$, of origin $O_{b}$ and axes $\mathbf{x}_{b}, \mathbf{y}_{b}$ and $\mathbf{z}_{b}$. The pose is composed by the vector of the Cartesian coordinates of the platform Centre of Mass $(\mathrm{CoM}), \mathbf{t}$, and the vector $\Phi=[\phi, \theta, \psi]^{\mathrm{T}}$ containing the roll, pitch and yaw angles, $\phi, \theta$ and $\psi$, around the axes $\mathbf{z}_{b}, \mathbf{y}_{b}$ and $\mathbf{x}_{b}$, respectively.

The cable connection points on the base, the so-called exit points, $A_{i}, i=1, \ldots, m$, are defined, with respect to $\mathscr{F}_{b}$, through the Cartesian coordinate vectors $\mathbf{a}_{i}^{b}, i=1, \ldots, m$. The platform-cable connection points, $B_{i}, i=1, \ldots, m$, are defined, with respect to a local reference frame $\mathscr{F}_{p}$, through the Cartesian coordinate vectors $\mathbf{b}_{i}^{p}, i=1, \ldots, m$. This local reference frame, of origin $O_{p}$ and axes $\mathbf{x}_{p}, \mathbf{y}_{p}$ and $\mathbf{z}_{p}$, is attached to the mobile platform.

\section{A. GEOMETRIC MODEL}

The geometric model of the robot consists of the chain closure equations for the cable vectors, $\mathbf{l}_{i}^{b}, i=1, \ldots, m$. Those vectors can be defined, with respect to $\mathscr{F}_{b}$, as:

$$
\mathbf{l}_{i}^{b}=\mathbf{a}_{i}^{b}-\mathbf{t}-\mathbf{R} \mathbf{b}_{i}^{p}, \quad i=1, \ldots, m
$$

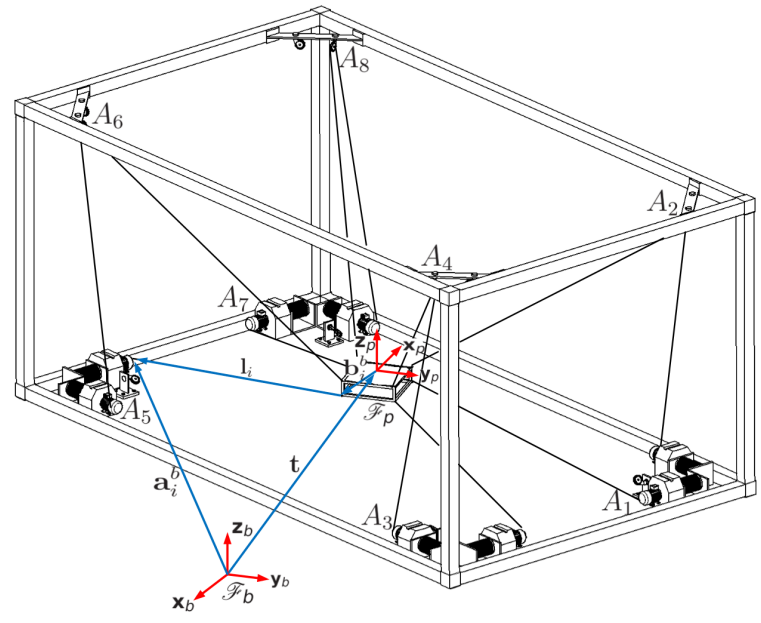

Fig. 2. CDPR geometric description.

where $\mathbf{R}$ denotes the orientation matrix of the platform:

$$
\begin{aligned}
\mathbf{R} & =\mathbf{R}_{z}(\phi) \mathbf{R}_{y}(\theta) \mathbf{R}_{x}(\psi)= \\
& =\left[\begin{array}{ccc}
c \phi c \theta & c \phi s \theta s \psi-s \phi c \psi & c \phi s \theta c \psi+s \phi s \psi \\
s \phi c \theta & s \phi s \theta s \psi+c \phi c \psi & s \phi s \theta c \psi-c \phi s \psi \\
-s \theta & c \theta s \psi & c \theta c \psi
\end{array}\right]
\end{aligned}
$$

The corresponding unit vectors are defined, with respect to $\mathscr{F}_{b}$, as:

$$
\mathbf{d}_{i}^{b}=\frac{\mathbf{l}_{i}^{b}}{\left\|\mathbf{l}_{i}^{b}\right\|_{2}}, \quad i=1, \ldots, m
$$

The geometric model of a generic CDPR is shown in Fig. 2.

\section{B. STATIC MODEL}

The static equilibrium of the CDPR mobile platform is described by the following equation:

$$
\mathbf{W} \boldsymbol{\tau}+\mathbf{w}_{e}=0
$$

where $\boldsymbol{\tau}=\left[\tau_{1}, \ldots, \tau_{m}\right]$ is the vector collecting all the cable tensions; $\mathbf{W}$ is the wrench matrix, whose columns are composed of the unit wrenches $\hat{\mathbf{w}}_{i}$ exerted by the cables on the platform:

$$
\mathbf{W}=\left[\begin{array}{cccc}
\mathbf{d}_{1}^{b} & \mathbf{d}_{2}^{b} & \ldots & \mathbf{d}_{m}^{b} \\
\mathbf{R b}_{1}^{p} \times \mathbf{d}_{1}^{b} & \mathbf{R b}_{2}^{p} \times \mathbf{d}_{2}^{b} & \ldots & \mathbf{R b}_{m}^{p} \times \mathbf{d}_{m}^{b}
\end{array}\right]
$$

$\mathbf{w}_{e}$ is the external wrench acting on the platform:

$$
\mathbf{w}_{e}=\left[\mathbf{f}^{\mathrm{T}}, \mathbf{m}^{\mathrm{T}}\right]^{\mathrm{T}}=\left[f_{x}, f_{y}, f_{z}, m_{x}, m_{y}, m_{z}\right]^{\mathrm{T}}
$$

The components of the force vector, $\mathbf{f}$, as well as the components of the moment vector, $\mathbf{m}$, are bounded as follows:

$$
\begin{gathered}
f_{\min } \leq f_{x}, f_{y}, f_{z} \leq f_{\max } \\
m_{\min } \leq m_{x}, m_{y}, m_{z} \leq m_{\max }
\end{gathered}
$$

In the following section, we will consider only fully constrained CDPRs. Hence, the inverse static problem is under-constrained and can be defined as:

$$
\boldsymbol{\tau}=\boldsymbol{\tau}_{n}+\boldsymbol{\tau}_{0}=\mathbf{W}^{\dagger} \mathbf{w}_{e}+\lambda \mathbf{n} \quad \boldsymbol{\tau} \geq \mathbf{0}
$$


where $\mathbf{W}^{\dagger}$ denotes the Moore-Penrose generalized inverse of $\mathbf{W}, \lambda \in \mathbb{R}$ and $\mathbf{n}$ is a vector in the null space of $\mathbf{W}$ [21]. A possible solution to (9) can be found by minimizing the 2-norm of the cable tension vector, $\|\boldsymbol{\tau}\|_{2}$.

\section{ELASTO-STATIC MODEL}

In order to quantify the stiffness of the system, an elastostatic model is used:

$$
\delta \mathbf{w}_{e}=\mathbf{K} \delta \mathbf{p}
$$

where the matrix $\mathbf{K}$ defines the relation between an infinitesimal change in the external wrench, $\delta \mathbf{w}_{e}$, and the corresponding infinitesimal change in the pose of the CDPR platform, $\delta \mathbf{p}=\left[\delta \mathbf{t}^{\mathrm{T}}, \delta \Phi^{\mathrm{T}},\right]^{\mathrm{T}}$. The reader is referred to [22] for a complete description of the stiffness matrix $\mathbf{K}$.

\section{THE TECHNICAL RECONFIGURATION PROCEDURE}

This section focuses on the technical description of the reconfiguration while section IV describes the procedure to be used in order to select the optimal configurations and their reconfiguration order. The core of the proposed reconfiguration algorithm, based on a Dijkstra's algorithm, is detailed in Section IV-B. In this paper, we mean by reconfiguration that the RCDPR is able to change the locations of its cable exit points. For some RCDPRs the choice is limited to a given number of reconfigurations: this is the typical case of a RCDPR whose cables can be connected to a grid of exit points, e.g. as illustrated in [22]. Other RCDPRs have at disposal an infinite number of configurations: this is the typical case of a RCDPR whose exit points are sliding on rails or along linear guides, e.g. as proposed in [15]. The RCDPRs analysed in this paper are restricted to the first category.

When the working environment is not cluttered, and the ground is free, it is possible to perform the reconfiguration on the ground. The CDPR platform is moved from the current pose to a home position on the ground. Then, when no external wrench is applied on the platform and the cables are slack, the cables can be disconnected easily from the platform. Once the exit points are reconfigured, the cables can be attached again to the platform, and the platform can be moved to a desired working pose.

The previous strategy cannot always be applied. For instance, a cluttered environment may not permit to put the CDPR platform in a home position on the ground. In the strategy proposed hereafter the platform of the CDPR should not or only slightly change its pose during the reconfiguration:

a. The robot is located at the desired reconfiguration pose.

The current pose of the robot is denoted hereafter as $\mathcal{C}_{a}$.

The cables balance the weight of the platform and the external wrenches applied on the platform, according to the static equilibrium equation $\mathbf{W}_{a} \boldsymbol{\tau}_{a}+\mathbf{w}_{e}=\mathbf{0}$.

b. A new set of cables are connected to the proper exit point locations, according to the new desired configuration, defined hereafter as $\mathcal{C}_{a^{\prime}}$. The other extremities of these

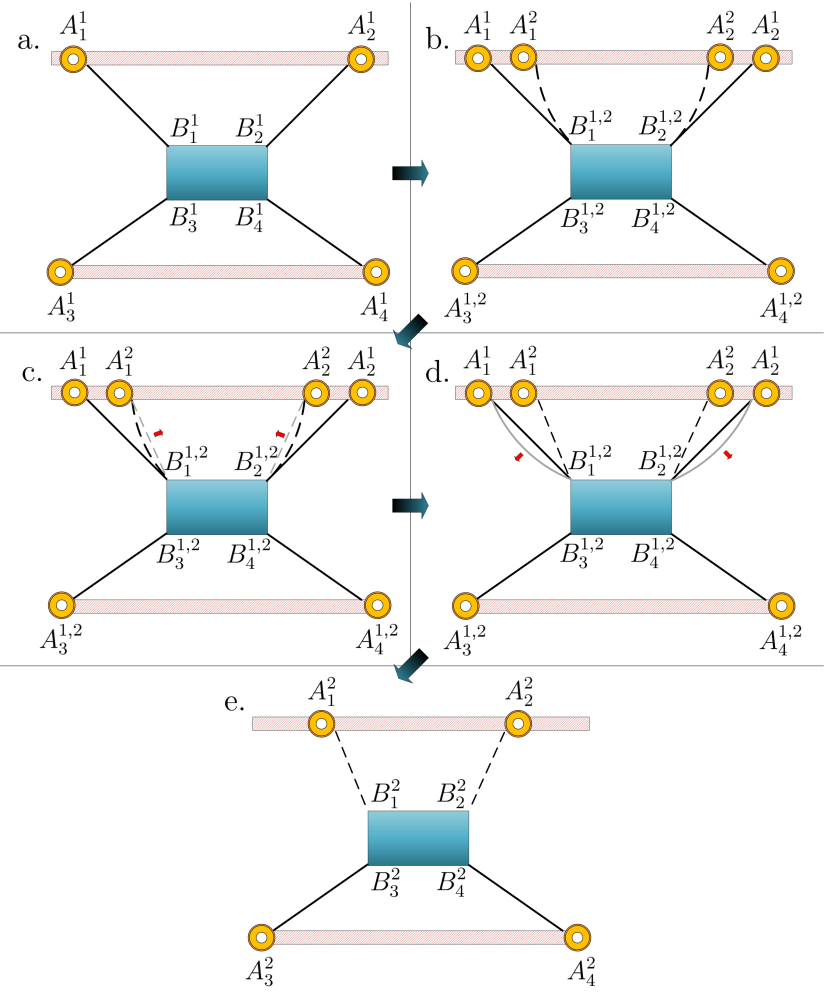

Fig. 3. Example of reconfiguration procedure for a 4-cable suspended planar robot. The continuous lines represent the cable associated to configuration $\mathcal{C}_{a}$; the dashed lines represent the cables associated exclusively to configuration $\mathcal{C}_{a^{\prime}}$. In this example only 2 cables are reconfigured.

cables are connected to the corresponding attachment points on the platform. These cables are initially slack and they are coiled in such a way to be "just tensed", their lengths being adjusted to the platform pose.

c. In order to be detached from the platform, the cables belonging to $\mathcal{C}_{a}$, but not to $\mathcal{C}_{a^{\prime}}$, should be slack. These cables are gradually uncoiled in such a way that the external wrenches are transferred to the cables belonging to $\mathcal{C}_{a^{\prime}}$.

d. When the cables belonging exclusively to configuration $\mathcal{C}_{a}$ are slack, they can be detached. The RCDPR is now ready to proceed with the task execution.

A simple example of this procedure is given in Fig. 3.

\section{THE RECONFIGURATION ALGORITHM}

In general, given a specific RCDPR, it is possible to select a combination of configurations to complete a given task. The selection can be performed with respect to one or more criteria, according to the algorithm presented hereafter and illustrated in Fig. 4.

1) The user defines the geometry of the RCDPR and provides the list of all the $n_{\mathcal{C}}$ possible reconfigurations.

2) The user defines the task characteristics, including: the desired path of the platform $\operatorname{CoM}, \mathcal{P}$, discretized into $n_{p}$ points according to the user specifications, $\mathcal{P}=\left\{P_{i}, \quad i=1, \ldots, n_{p}\right\} ;$ the bounds on the external wrenches applied to the platform, $f_{\min }, f_{\max }, m_{\min }$, $m_{\max }$. 


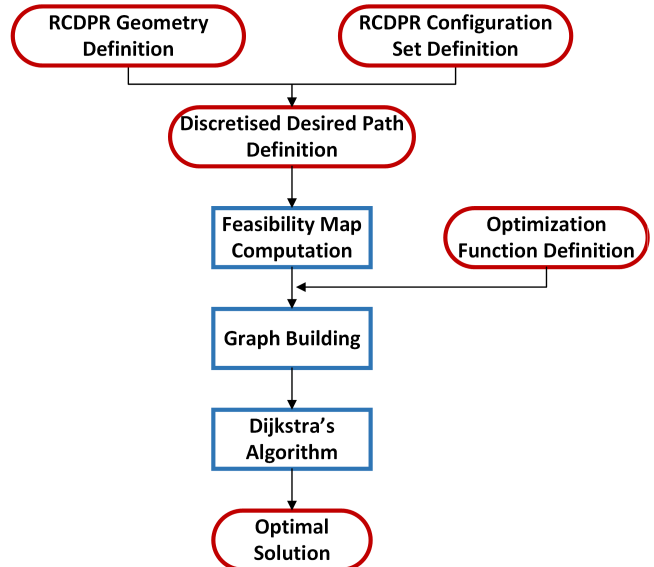

Fig. 4. Scheme of the reconfiguration algorithm.

3) The algorithm computes a feasibility map. For each point $P_{i} \subset \mathcal{P}$, all the $n_{\mathcal{C}}$ configurations should be tested, in order to verify their feasibility. For a given configuration $\mathcal{C}_{j}$, the pose associated to the $i$-th point of the desired path, $P_{i, j}$, will be feasible, $P_{i, j}=1$, if it satisfies a list of $n_{\phi}$ constraints, $\phi_{i, j, k}, k=1, \ldots, n_{\phi}$, specified by the user:

$$
P_{i, j}=1 \quad \Longleftrightarrow \quad \sum_{k=1}^{n_{\phi}} \phi_{i, j, k}=0
$$

where:

$$
\left\{\begin{array}{l}
\phi_{i, j, k}=0 \quad \Longleftrightarrow \quad \text { the constraint is satisfied } \\
\phi_{i, j, k}=1 \quad \Longleftrightarrow \quad \text { the constraint is not satisfied }
\end{array}\right.
$$

The following constraints have been taken into account in this paper:

- The moving-platform pose is wrench feasible. The cables should be able to balance any possible external wrench $\mathbf{w}_{e}$, according to the ranges specified in (7) and (8). This set of required external wrenches consists of an hyperectangle $\left[\mathbf{w}_{e}\right]_{r}$. Hence, the configuration is wrench feasible if the required external wrenches are included inside the zonotope of the available wrenches, $\left[\mathbf{w}_{e}\right]_{a}$, as follows:

$$
\forall \mathbf{w}_{e} \in[\mathbf{w}]_{r}, \exists \boldsymbol{\tau} \in[\boldsymbol{\tau}] \text { s.t. }\left\{\begin{array}{l}
\mathbf{w}_{e} \in[\mathbf{w}]_{a} \\
\mathbf{W} \boldsymbol{\tau}+\mathbf{w}_{e}=0
\end{array}\right.
$$

This condition can be translated into a set of inequalities, according to [23]:

$$
\mathbf{C w}_{e} \leq \mathbf{d}, \quad \forall \mathbf{w}_{e} \in[\mathbf{w}]_{r}
$$

- No cable interference occurs. This condition is verified by measuring the distance between each pair of cables, e.g. with the procedure explained in [24]. The interference between the $i$-th cable and a.

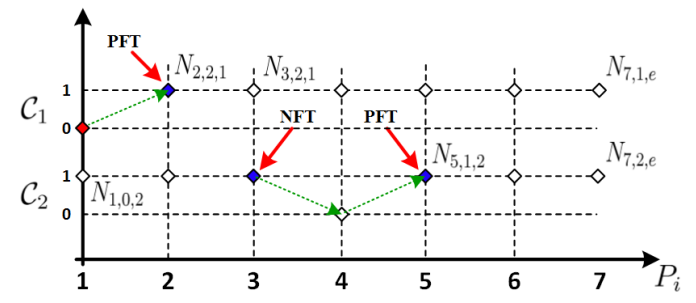

b.

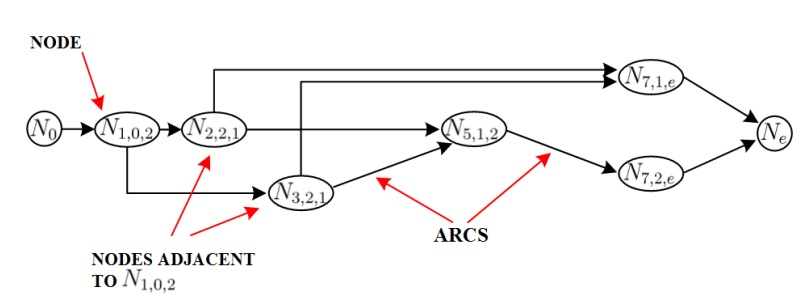

Fig. 5. Example of a feasibility map and the related graph.

the $j$-th cable does not occur when the distance $d_{i, j}^{c c}$ is greater than the diameter of the cables, $\phi_{c}$, i.e.,

$$
d_{i, j}^{c c} \geq \phi_{c} \quad \forall i, j=1, \ldots, m, \quad i \neq j
$$

- No cable-environment collision occurs. The verification is performed using the same principle as the cable interference, being given that the obstacles are discretized into several facets.

- The components of the positioning error due to the RCDPR cable elasticity, $\delta \mathbf{t}=\left[\delta \mathbf{t}_{x}, \delta \mathbf{t}_{y}, \delta \mathbf{t}_{z}\right]$, are lower than then corresponding components of a threshold vector $\delta \mathbf{t}_{c}=\left[\delta \mathbf{t}_{x, c}, \delta \mathbf{t}_{y, c}, \delta \mathbf{t}_{z, c}\right]$ defined by the user.

4) The algorithm builds a graph from the feasibility map.

5) The Dijkstra's algorithm solves the optimization problem according to the criteria specified by the user.

The details of Step 4. and Step 5. are given in the following subsections.

An example of a simple feasibility map is provided in Fig. 5a. The feasibility map illustrated in Fig. 5a. represents the feasible poses associated to each configuration. In this example, two configurations are taken into account, $\mathcal{C}_{1}$ and $\mathcal{C}_{2}$. The poses, defined as $P_{i, j}, i=1, \ldots, 7, j=1,2$, are equal to 1 when they are feasible and equal to 0 when they are not. We can notice that pose $P_{3,2}$ is feasible while $P_{4,2}$ is infeasible, i.e., the configuration $\mathcal{C}_{2}$ changes from a feasible to an infeasible condition. On the contrary, between the poses $P_{4,2}$ and $P_{5,2}$, configuration $\mathcal{C}_{2}$ is subjected to a transition from an infeasible to a feasible condition. In both cases we will talk about Feasibility Transitions (FT). The FTs in the feasibility map of Fig. 5a are represented by three green arrows. When a FT occurs between the poses $P_{i-1, j}$ and $P_{i, j}$, in such a way that $P_{i-1, j}$ is infeasible and $P_{i, j}$ is feasible, we will refer to the pose $P_{i, j}$ as a Positive Feasibility Transition (PFT). Thus:

$$
P_{i, j} \text { is a PFT if }\left\{\begin{array}{l}
P_{i, j}=1 \\
P_{i-1, j}=0
\end{array}\right.
$$


On the contrary, when a FT occurs between the points $P_{i, j}$ and $P_{i+1, j}$, in such a way that $P_{i, j}$ is feasible and $P_{i+1, j}$ is infeasible, we will refer to the point $P_{i, j}$ as a Negative Feasibility Transition (NFT). Thus:

$$
P_{i, j} \text { is a NFT if }\left\{\begin{array}{l}
P_{i, j}=1 \\
P_{i+1, j}=0
\end{array}\right.
$$

\section{A. GRAPH NODES}

In order to find the best reconfiguration strategy, using Dijkstra's algorithm, it is necessary to build a proper graph. A graph is a set of nodes connected to each others through arcs. The nodes of the graph represent the reconfigurations that the RCDPR can perform. Hereafter, the nodes will be denoted as $N_{i, j, k}$, where the indices $i, j$ and $k$ are defined as follows:

- The index $i$ represents the point $P_{i}$ to which the node is associated.

- The index $j$ represents the starting configuration $\mathcal{C}_{j}$.

- The index $k$ represents the final configuration $\mathcal{C}_{k}$ to which the RCDPR is reconfigured, starting from the configuration $\mathcal{C}_{j}$.

The nodes connected by arcs will be defined hereafter as adjacent nodes. Two nodes $N_{i, j, k}$ and $N_{i^{\prime}, j^{\prime}, k^{\prime}}$, with $i^{\prime}>i$ and $k=j^{\prime}$, are adjacent when all the poses between $P_{i, k}$ and $P_{i^{\prime}, k}$ are feasible: $P_{i^{\prime \prime}, k}=1, \quad \forall i^{\prime \prime}$ s.t. $i \leq i^{\prime \prime} \leq i^{\prime}$.

\section{B. GRAPH BUILDING}

The graph to be searched with the Dijkstra's algorithm is generated by analyzing the feasibility map. The procedure, illustrated in Fig. 6, consists of the following steps:

1) The first node, $N_{0}$, is an empty virtual node. Its position along the desired path is 0 and it is not associated to any configuration. Its adjacent nodes, $N_{1,0, j}$, are the ones associated to the first point, $P_{1}$, of the desired path. A node $N_{1,0, j}$ is created if and only if the pose $P_{1, j}$ is feasible.

2) The intermediate nodes are obtained by analyzing each point of the desired path. For a given configuration, $\mathcal{C}_{j}$, the algorithm verifies the presence of a FT at pose $P_{i, j}$. When a NFT is encountered at $P_{i, j}$, the RCDPR can (and should) be reconfigured in such a way to reach any configuration $\mathcal{C}_{k}$ that is feasible at $P_{i, k}$ and $P_{i+1, k}$. Thus, a node $N_{i, j, k}$ is created if $P_{i, j}$ is a NFT, $P_{i, k}=1$ and $P_{i+1, k}=1$. When $P_{i, j}$ is a PFT, provided that $P_{i+1, j}=1$, the RCDPR can be reconfigured in such a way to reach the configuration $\mathcal{C}_{j}$ starting from any configuration $\mathcal{C}_{k}$ that is feasible at $P_{i, k}$. Thus, a node $N_{i, k, j}$ is created if $P_{i, j}$ is a PFT, $P_{i, k}=1$ and $P_{i+1, j}=1$. It is worth noting that, in all the cases, a node is created when the initial and the final configurations are both feasible.

3) The final node, $N_{f}$, is an empty virtual node. Its position is at the end of the desired path and it is not associated to any configuration. Its adjacent nodes, $N_{n_{P}, j, f}$, are associated to the last point, $P_{n_{P}}$, of the desired path.

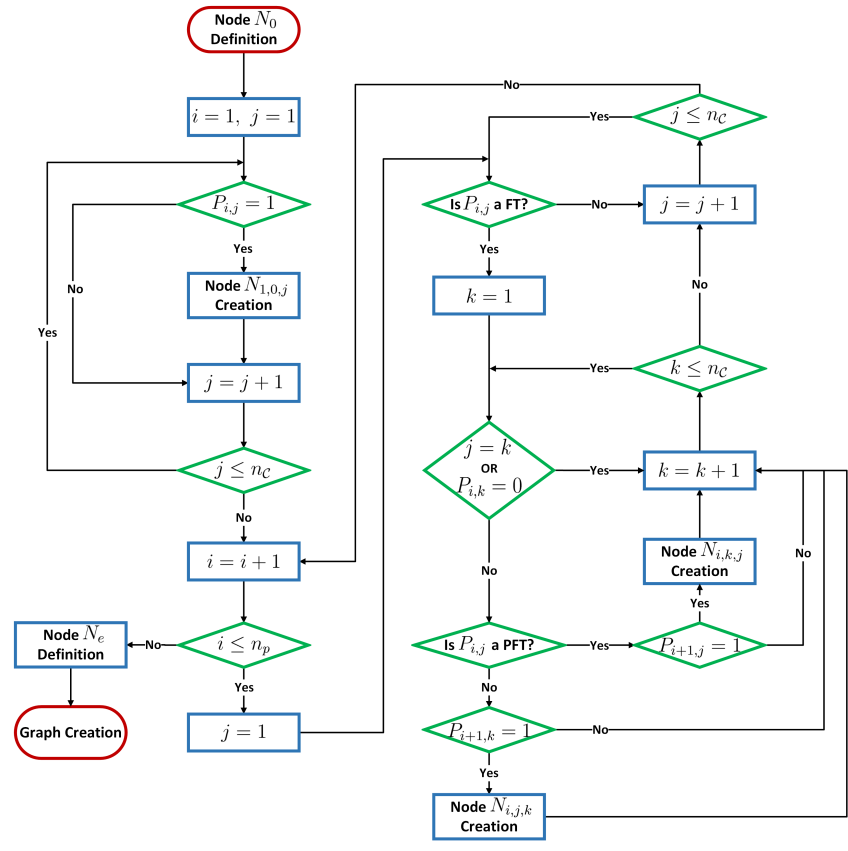

Fig. 6. Scheme of the algorithm used to build the graph for the Dijkstra's algorithm.

They are created if and only if the poses $P_{n_{P}, j}$ are feasible.

The arcs are then created between the couples of adjacent nodes, the latter being defined in Section IV-A. Once the graph is built, the Dijkstra's algorithm searches the graph looking for the minimum cost path connecting nodes $N_{0}$ and $N_{f}$. The cost associated to each arc of the graph is defined according to the procedure described in Section IV-C.

\section{THE ARC COST FUNCTION}

Let the $q$-th arc connect the nodes $N_{i, j, k}$ and $N_{i^{\prime}, j^{\prime}, k^{\prime}}$. Its cost function $\Phi_{q}$ is defined according to $n_{\lambda}$ criteria, $\lambda_{t, q}, t=1, \ldots, n_{\lambda}$, and is formulated as follows:

$$
\Phi_{q}=\sum_{t=1}^{n_{\lambda}} \lambda_{t, q} \mu_{t}, \quad \mu_{t} \in[0,1], \quad \sum_{t=1}^{n_{\lambda}} \mu_{t}=1
$$

The weighting factors $\mu_{t}, t=1, \ldots, n_{\lambda}$, are defined according to the priority assigned to each criterion. The criteria $\lambda_{t, q}$ are bounded between 0 and 1 . Hereafter, three criteria are considered:

$$
\begin{aligned}
\lambda_{1, q} & =1 \\
\lambda_{2, q} & =\frac{n_{c, q}}{\frac{m}{\left\|\delta \mathbf{t}_{q}\right\|_{2}}} \\
\lambda_{3, q} & =\frac{\left\|\delta \mathbf{t}_{c}\right\|_{2}}{\| x}
\end{aligned}
$$

$\lambda_{1, q}$ is a constant cost function equal to 1 used in order to count the number of reconfigurations performed during the task execution. $\lambda_{2, q}$ is proportional to the number of cables, $n_{c, q}$, to be reconfigured when the RCDPR passes from configuration $\mathcal{C}_{j}^{\prime}$ to configuration $\mathcal{C}_{k}^{\prime} ; \lambda_{3, q}$ is proportional to 
the mean of the norm of the positioning error:

$$
\overline{\left\|\delta \mathbf{t}_{q}\right\|_{2}}=\frac{\sum_{i^{\prime \prime}=i}^{i^{\prime}}\left\|\delta \mathbf{t}_{i^{\prime \prime}, k}\right\|_{2}}{i^{\prime}-i}
$$

where $\left\|\delta \mathbf{t}_{i^{\prime \prime}, k}\right\|_{2}$ is the norm of the cable tension vector measured at the pose $P_{i^{\prime \prime}}$ with respect to the configuration $\mathcal{C}_{k}$. In (20), $\overline{\left\|\delta \mathbf{t}_{q}\right\|_{2}}$ is normalized with respect to the norm of the critical precision threshold vector $\left\|\delta \mathbf{t}_{c}\right\|_{2}$.

The proposed cost functions aim at improving the efficiency of the RCDPR. By minimizing the arc cost associated to $\lambda_{1, q}$, the algorithm aims at reducing the total number of reconfigurations $n_{r}$ required to complete the task. The minimization of the arc cost associated to $\lambda_{2, q}$ corresponds to the minimization of the total number $n_{c}$ of cable detaching-attaching operations required to complete the task, and consequently to the minimization of the reconfiguration set-up time. By minimizing the arc cost associated to $\lambda_{3, q}$, the average of the RCDPR stiffness is increased and the positioning error is reduced.

\section{CASE STUDIES}

\section{A. First Case Study}

A first case study is considered in order to illustrate the algorithm presented in Section. IV. A closed path $\mathcal{P}$, discretized in 164 points, $P_{i}, i=1, \ldots, 164$, has to be followed by the platform CoM. The path, lying in the plane $\left(O, \mathbf{x}_{b}, \mathbf{y}_{b}\right)$, is illustrated in Fig. 7. The orientation of the platform is constant, with zero roll, pitch and yaw angles.

An obstacle is included in the environment. It consists of a square of side length equal to $1 \mathrm{~m}$. The obstacle is centered at the origin of the reference frame $\mathscr{F}_{b}$ as shown in Fig. 7.

The RCDPR under study is composed of four cables. The cable exit points, $A_{i}, i=1, \ldots, 4$ can be reconfigured, according to the following parametrization:

$$
\begin{aligned}
\mathbf{a}_{1}^{b} & =\left[\begin{array}{ll}
u_{1}+u_{3}, & u_{2}
\end{array}\right]^{\mathrm{T}} \\
\mathbf{a}_{2}^{b} & =\left[\begin{array}{ll}
-u_{1}+u_{3}, & u_{2}
\end{array}\right]^{\mathrm{T}} \\
\mathbf{a}_{3}^{b} & =\left[\begin{array}{ll}
-u_{1}+u_{3}, & -u_{2}
\end{array}\right]^{\mathrm{T}} \\
\mathbf{a}_{4}^{b} & =\left[\begin{array}{ll}
u_{1}+u_{3}, & -u_{2}
\end{array}\right]^{\mathrm{T}}
\end{aligned}
$$

The parameters $u_{1}$ and $u_{2}$ represent half of the distance between the exit points along $\mathbf{x}_{b}$ and $\mathbf{y}_{b}$, respectively. $u_{3}$ is the offset of the cables connection point positions along $\mathbf{x}_{b}$. The robot is symmetric with respect to $\mathbf{x}_{b}$, as shown in Fig. 7. Four configurations have been considered in this case study. Their parameters are represented in Tab. I.

The dimensions of the platform have been set to $0.2 \mathrm{~m}$ for the width, $w_{p}$, and $0.3 \mathrm{~m}$ for the height, $h_{p}$. The Cartesian coordinates of points $B_{i}, i=1, \ldots, 4$, expressed in the moving-platform frame $\mathscr{F}_{p}$ are the following:

$$
\begin{aligned}
\mathbf{b}_{1}^{p} & =\left[w_{p} / 2, h_{p} / 2\right]^{\mathrm{T}} \\
\mathbf{b}_{2}^{p} & =\left[\begin{array}{ll}
-w_{p} / 2, & h_{p} / 2
\end{array}\right]^{\mathrm{T}} \\
\mathbf{b}_{3}^{p} & =\left[-w_{p} / 2,-h_{p} / 2\right]^{\mathrm{T}} \\
\mathbf{b}_{4}^{p} & =\left[w_{p} / 2,-h_{p} / 2\right]^{\mathrm{T}}
\end{aligned}
$$

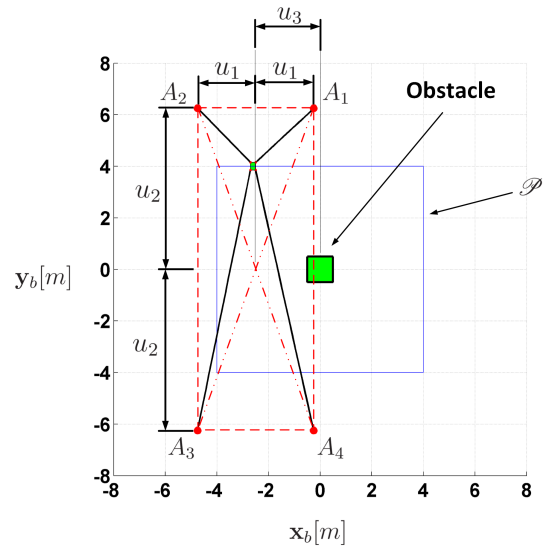

Fig. 7. First case study and description of the exit point parametrization for the RCDPR.

TABLE I

Possible CONFIGURATIONS OF THE FIRST CASE STUdY.

\begin{tabular}{cccc}
\hline \hline Configuration & $u_{1}$ & $u_{2}$ & $u_{3}$ \\
\hline $\mathcal{C}_{1}$ & 2.25 & 6.25 & 2.50 \\
$\mathcal{C}_{2}$ & 4.00 & 7.75 & -1.00 \\
$\mathcal{C}_{3}$ & 4.00 & 7.00 & -0.25 \\
$\mathcal{C}_{4}$ & 2.25 & 6.25 & -2.50 \\
\hline \hline
\end{tabular}

The properties of the cables have been selected as follows: the Young Modulus, $E$, is equal to $100 \mathrm{GPa}$; their diameter $\phi_{c}$ is equal to $4 \mathrm{~mm}$; their stiffness coefficient, $k_{i}$, is equal to $252 \mathrm{KN} / \mathrm{m}$; the maximum allowed tension in the cables, $\tau_{\max }$, is equal to $34950 \mathrm{~N}$. A safety coefficient $\eta=5$ has been taken into account such that:

$$
0<\tau_{i} \leq \frac{\tau_{\max }}{\eta}, \quad \forall i=1, \ldots, 4
$$

The platform can be subjected to an external wrench, $\mathbf{w}_{e}$. We assume that the components of $\mathbf{w}_{e}$ are bounded as follows:

$$
\begin{aligned}
& -500 \mathrm{~N} \leq f_{x}, f_{y} \leq 500 \mathrm{~N} \\
& -7.5 \mathrm{~N} \leq m_{z} \leq 7.5 \mathrm{~N}
\end{aligned}
$$

where $f_{x}$ and $f_{y}$ stand for the external force components along $\mathbf{x}_{b}$ and $\mathbf{y}_{b}$ axes, respectively. $m_{z}$ is the external moment about the $\mathbf{z}_{b}$ axis.

The first analysis has been performed by optimizing the total number of reconfigurations, $n_{r}$, according to the weighting factors $\mu_{1}=1, \mu_{2}=\mu_{3}=0$. The solution proves that four reconfigurations are sufficient to complete the proposed task. The optimal result is shown in Fig. 8. The feasibility map has been built by analyzing the constraints applied on each point of the trajectory namely, wrench feasibility, cable interferences and obstacle collisions.

For instance, configuration $\mathcal{C}_{4}$ is the unique feasible configuration at point $P_{1}$ of the desired path. The NFT at point $P_{16}$ requires a change of configuration, from $\mathcal{C}_{4}$ to $\mathcal{C}_{3}$. When a new NFT appears at point $P_{28}$, the RCDPR adopts the configuration $\mathcal{C}_{1}$. A PFT appears at point $P_{97}$. 


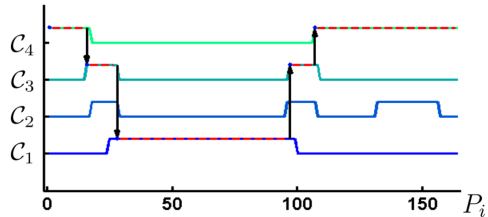

Fig. 8. First case study feasibility map and optimal solution with respect the minimization of $n_{r}$

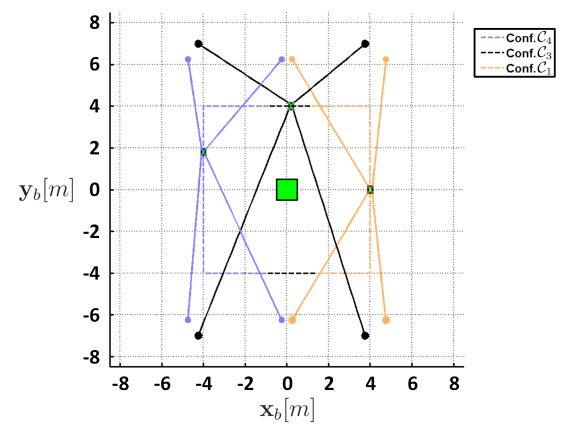

Fig. 9. First case study optimal RCDPR configurations, selected by the algorithm in order to complete the task with respect to the minimum number of configurations $n_{r}$.

The RCDPR returns to the configuration $\mathcal{C}_{3}$ and, later on, to the configuration $\mathcal{C}_{4}$, following the desired path till its end.

The optimal configurations required to complete the task are shown in Fig. 9.

A second optimization has been tested, aiming at minimizing the positioning error of the platform due to the cable elasticity, according to the weighting factors $\mu_{1}=\mu_{2}=0, \mu_{3}=1$. The result is similar to the one obtained by minimizing $n_{r}$. In this specific case, the first reconfiguration is performed at $P_{18}$. The RCDPR passes from the configuration $\mathcal{C}_{4}$ to the configuration $\mathcal{C}_{2}$ as shown in Fig. 10.

Both the optimal solutions analyzed in this case study are represented in the graph shown in Fig. 11. The red arrows indicate the path obtained by minimizing $n_{r}$. The blue arrows describe the path generated by minimizing the platform positioning error. The blue optimal path leads to a reduction of the positioning error, with respect to the red optimal path, of almost 18\%: the total cost of the red path, in terms of positioning error, is equal to 1.7663 while the cost associated to the blue path is equal to 1.4545 .

\section{B. Second Case Study}

A second case study is now considered. The requirements in terms of desired path and external wrenches are similar to the previous case, as well as the desired orientation of the platform. The platform geometry and the cable properties are also identical. However, the number of obstacles has been increased to three, as illustrated in Fig. 12.

The robot parametrization is defined by (22-25) and (26-29). The RCDPR under study can take nine configurations. Their parameters are given in Tab. II.

The computation has been performed by assigning the same weighting factor to all the criteria composing the cost

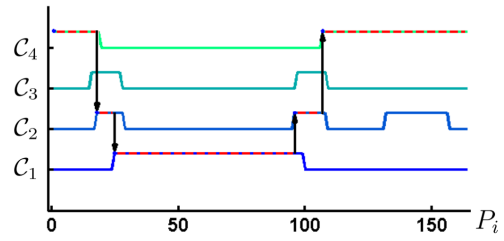

Fig. 10. First case study optimal solution with respect the minimization of the positioning error $\delta \mathbf{t}$.

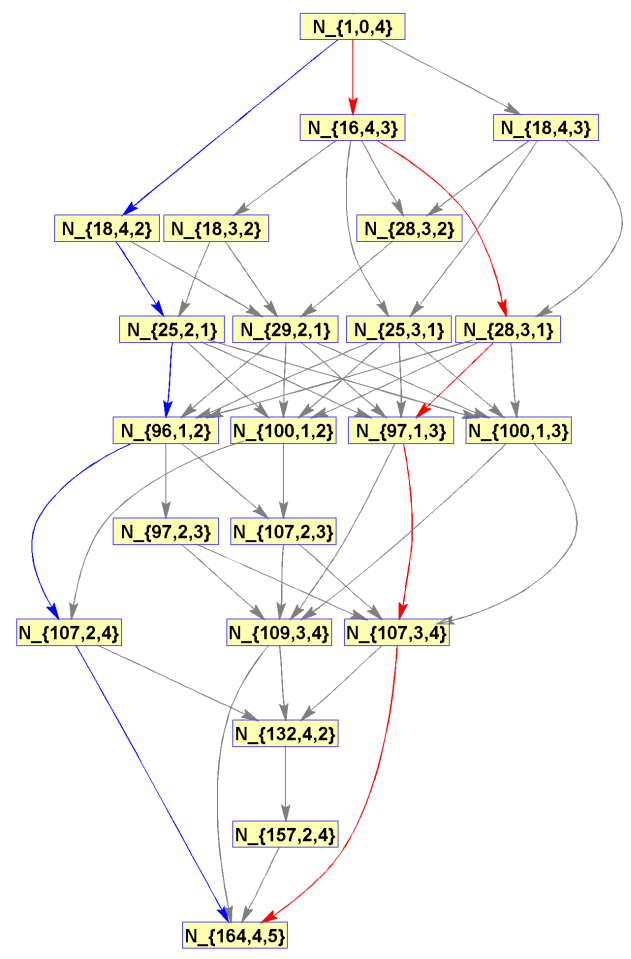

Fig. 11. Graph of the two optimization problems of the first case study.

functions: $\mu_{i}=\frac{1}{3}, i=1, \ldots, 3$. It turns out that the number of required configurations is equal to eight, as shown in Fig. 13.

\section{CONCLUSIONS AND FUTURE WORK}

The research work presented in this paper deals with the selection of an optimal reconfiguration strategy for RCDPRs. The proposed strategy is based on the Dijkstra's algorithm and aims notably at minimizing the number of reconfigurations, as well as the reconfiguration set-up time. The proposed algorithm permits the optimization of other characteristics of the RCDPR by means of a cost function depending on multiple criteria.

Two planar case studies have been used in order to validate the proposed reconfiguration strategy. The results show that the algorithm is able to compute the optimal solutions with respect to a set of configurations proposed by the user. The results can be extended to three-dimensional problems.

The algorithm computation time is quite satisfactory when the RCDPR has a limited number of possible configurations. Each of the previous simulations required a graph resolution 


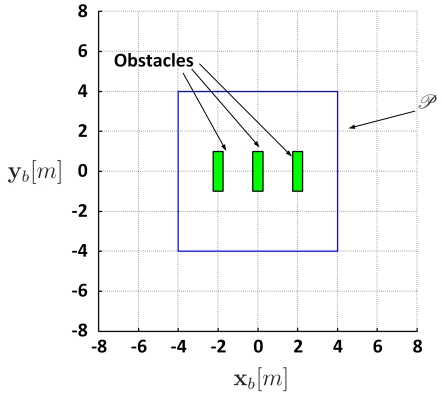

Fig. 12. Second case study.

TABLE II

POSSIBLE CONFIGURATIONS OF THE SECOND CASE STUDY.

\begin{tabular}{cccc}
\hline \hline Configuration & $u_{1}$ & $u_{2}$ & $u_{3}$ \\
\hline $\mathcal{C}_{1}$ & 1.25 & 4.75 & 3.50 \\
$\mathcal{C}_{2}$ & 2.75 & 6.25 & 2.00 \\
$\mathcal{C}_{3}$ & 3.25 & 7.50 & 2.75 \\
$\mathcal{C}_{4}$ & 3.25 & 7.75 & 3.25 \\
$\mathcal{C}_{5}$ & 2.50 & 5.50 & 0 \\
$\mathcal{C}_{6}$ & 3.25 & 7.75 & -3.25 \\
$\mathcal{C}_{7}$ & 3.25 & 7.50 & -2.75 \\
$\mathcal{C}_{8}$ & 2.75 & 6.25 & -2.00 \\
$\mathcal{C}_{9}$ & 1.25 & 4.75 & -3.50 \\
\hline \hline
\end{tabular}

time lower than $0.01 \mathrm{~s}$. However, the main limitation is related to the time needed to construct the feasibility map, especially when the algorithm has to deal with threedimensional RCDPRs which have a large number of possible configurations. Hence, further studies should be dedicated to the improvement of Step. 3) of the algorithm presented in Section IV.

\section{ACKNOWLEDGMENT}

This study is part of the CAROCA project managed by IRT Jules Verne (French Institute in Research and Technology in Advanced Manufacturing Technologies for Composite, Metallic and Hybrid Structures).

\section{REFERENCES}

[1] C. Gosselin, "Cable-driven parallel mechanisms: state of the art and perspectives," Mechanical Engineering Reviews, vol. 1, no. 1, pp. 117, 2014.

[2] SkyCam [Online]. Available: http://skycam.tv/.

[3] SpyderCam [Online]. Available: http://spydercam.com/.

[4] J. Albus, R. Bostelman, and N. Dagalakis, "The NIST spider, a robot crane," J. of Research of the Nat. Inst. of Standards and Technology, vol. 97 , no. 3, pp. 373-385, May 1992.

[5] A. Pott, C. Meyer, and A. Verl, "Large-scale assembly of solar power platns with parallel cable robots," in International Symposium on Robotics, 2010, pp. 1-6.

[6] C. Holland and D. Cannon, "Cable array robot for material handling," U.S. Patent 6826452, March 2002.

[7] G. Rosati, M. Andreolli, A. Biondi, and P. Gallina, "Performance of cable suspended robots for upper limb rehabilitation," in IEEE 10th International Conference on Rehabilitation Robotics (ICORR), 2007, pp. 385-392.

[8] P. Gallina, G. Rosati, and A. Rossi, "3-dof wire driven planar haptic interface," Journal of Intelligent and Robotic Systems, vol. 32, no. 1, pp. 23-36, 2001.

[9] J.-P. Merlet, "Kinematics of the wire-driven parallel robot marionet using linear actuators," in Proc. IEEE Int. Conf. on Robotics and Automation, Passadena, May, pp. 3857-3862.

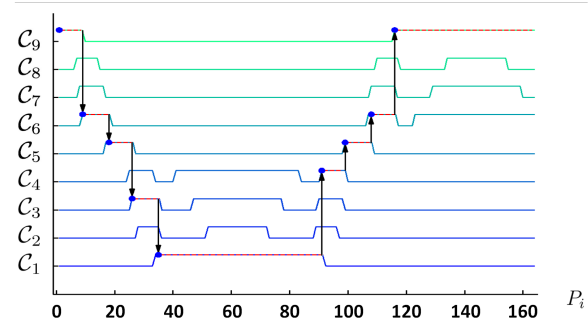

Fig. 13. Second case study feasibility map and optimal solution with respect the minimization of $n_{r}$.

[10] C. Gosselin, "Global planning of dynamically feasible trajectories for three-dof spatial cable-suspended parallel robots," Cable-Driven Parallel Robots, pp. 3-22, 2013.

[11] J.-D. Dechênes et al., "A cable-driven parallel mechanism for capturing object appearance from multiple viewpoints," in 6th International Conference on 3-D Digital Imaging and Modeling, Montréal, Canada, 2007.

[12] G. Rosati, D. Zanotto, and S. K. Agrawal, "On the design of adaptive cable-driven systems," Journal of Mechanisms and Robotics, vol. 3, May 2011.

[13] A. Zhou, C. P. Tang, and V. Krovi, "Analysis framework for cooperating mobile cable robots," in IEEE Int. Conf. on Robotics and Automation, RiverCentre, Saint Paul, MN, May 2012, pp. 3128-3133.

[14] J.-B. Izard, M. Gouttefarde, O. Tempier, and C. Baradat, "A reconfigurable robot for cable-driven parallel robotic research and industrial scenario proofing," in Cable-Driven Parallel Robots, 2013, vol. 12 , pp. 135-148.

[15] D.-Q. Nguyen, M. Gouttefarde, O. Company, and F. Pierrot, "On the analysis of large-dimension reconfigurable suspended cable-driven parallel robots," in IEEE International Conference on Robotics and Automation (ICRA), 2014, pp. 5728-5735.

[16] D.-Q. Nguyen and M. Gouttefarde, "Study of reconfigurable suspended cable-driven parallel robots for airplane maintenance," in IEEE International Conference on Intelligent Robots and Systems (IROS), 2014.

[17] CableBOT [Online]. Available: http://www.cablebot.eu/en/.

[18] E. W. Dikstra, "A note on two problems in connection with graphs," Numerische Mathematik, vol. 1, 1959.

[19] J. Siek, L.-Q. Lee, and A. Lumsdaine, The Boost Graph Library: User Guide and Refrence Manual. Addison Wesley, 2001.

[20] S. Caro, D. Chablat, and Y. Hu, "Algorithm for the actuation mode selection of the parallel manipulator navaro," in Proceeding of the ASME 2014 International Design Engineering Technical Conference \& Computers and Information in Engineering Conference IDETC/CIE 2014, Buffalo, NY, USA, Aug. 17-20 2014.

[21] R. Roberts, T. Graham, and T. Lippitt, "On the inverse kinematics, statics, and fault tolerance of cable-suspended robots," J. of Robotic Systems, vol. 15, no. 10, pp. 581-597, 1998.

[22] L. Gagliardini, S. Caro, M. Gouttefarde, P. Wenger, and A. Girin, "A reconfigurable cable-driven parallel robot for sandblasting and painting of large structures," in Cable-Driven Parallel Robots. Springer International Publishing, 2015, vol. 32, pp. 275-291.

[23] M. Gouttefarde and S. Krut, "Characterization of parallel manipulator available wrench set facets," in Advances in Robot Kinematics: Motion in Man and Machine, Piran, 2010, pp. 475-484.

[24] V. Lumelsky, "On fast computation of distance between line segments," Information Processing Letters, vol. 21, 1985. 